\title{
Unveiling the Mechanism of Liposome Formation Using the Graphene Liquid Cells
}

Surya Narayanan ${ }^{1}$, Emre Firlar ${ }^{1,2}$, Tara Foroozan ${ }^{2}$, Ramin Rojaee ${ }^{2}$, Dionna Bidny ${ }^{1}$, Reza Shahbazian Yassar $^{2}$ and Tolou Shokuhfar ${ }^{1,4}$

1. University of Illinois at Chicago, Department of Bioengineering, Chicago, USA.

2. University of Illinois at Chicago, Department of Industrial and Mechanical Engineering, Chicago, USA.

3. University of Illinois at Chicago, College of Dentistry, Chicago, USA.

Imaging soft biological materials have always been challenging owing to the low contrast and also weak structural integrity of the biomolecules under the electron beam. Conventional transmission electron microscopy (TEM) sample preparation involves staining and fixing which can introduce artifacts, that can alter the crucial information obtained from these samples. With in situ liquid microscopy, it is possible to image soft materials such as liposomes with higher contrast [1]. However, due to the thickness of the silicon nitride membranes used in liquid flow holders, certain vital information might be lost. Also, the ability of silicon to produce hydroxyl radicals and their interaction with the sample can diversify the dynamics of liposome formation [2]. Here, we use the graphene liquid cells (GLCs) to unveil the mechanism of liposome formation.

The formation of vesicles within the cells have attracted many biologists to explore and understand the mechanism, to mimic the functions outside the cell. Liposomes are one such man-made phospholipid vesicles. It has been widely studied due to its potential application in different fields including targeted drug delivery systems to deliver active drug molecules to the site of action. Although the applications have been widely explored, the mechanism of formation of liposomes are not well understood. There are many reasons that contribute to it: the current spectroscopy techniques and the conventional microscopy techniques do not have the temporal resolution to capture the events and the formation of the intermediate byproducts. The physical and the chemical methods adopted for the preparation of liposomes can also be important factors contributing to the uncertainty [3].

There are two proposed mechanisms in the literature which elucidate the series of events that could happen during the formation of liposome:1. Slicing of the bilayers to small pieces which forms individual liposomes 2. Formation of daughter vesicles as they sprout from the mother liposomes [4] as shown in figures $1 \mathrm{~A}$ and $1 \mathrm{~B}$.

The mechanism of liposome formation was explored by exposing the liposomes with certain electron dose using GLC-TEM. The bubble formation was observed and further, the formation of the daughter vesicles was observed as shown in Figure 1C. The samples were prepared by suspending the freshly prepared liposomes in water and further encapsulating the liposomes in GLCs as described by Wang et $a l, 2014$ [5]. The results obtained from this preliminary studies would open new avenues in the area of drug delivery systems and polymer chemistry [6].

References:

[1] Sarah M Hoppe, et al, Langmuir, 29 (2013), pp 9958.

[2] Cho Hoduk, et al, Nano Lett, 17 (2017), p. 414. 
[3] Danilo D. Lasic, Biochem.J. 256 (1988), p 1.

[4] D.D. Lasic, Journal of Liposome Research, 5 (1995), p 431.

[5] Wang C. et al, Advanced Materials 26 (2014), p.3410.

[6] The authors acknowledge funding from the National Science Foundation - CAREER award- Grant No- DMR 1564950. Prof. Tolou Shokuhfar is acknowledged for her many useful discussions and contribution to this work. This work made use of instruments in the Electron Microscopy Service (Research Resources Center, UIC).

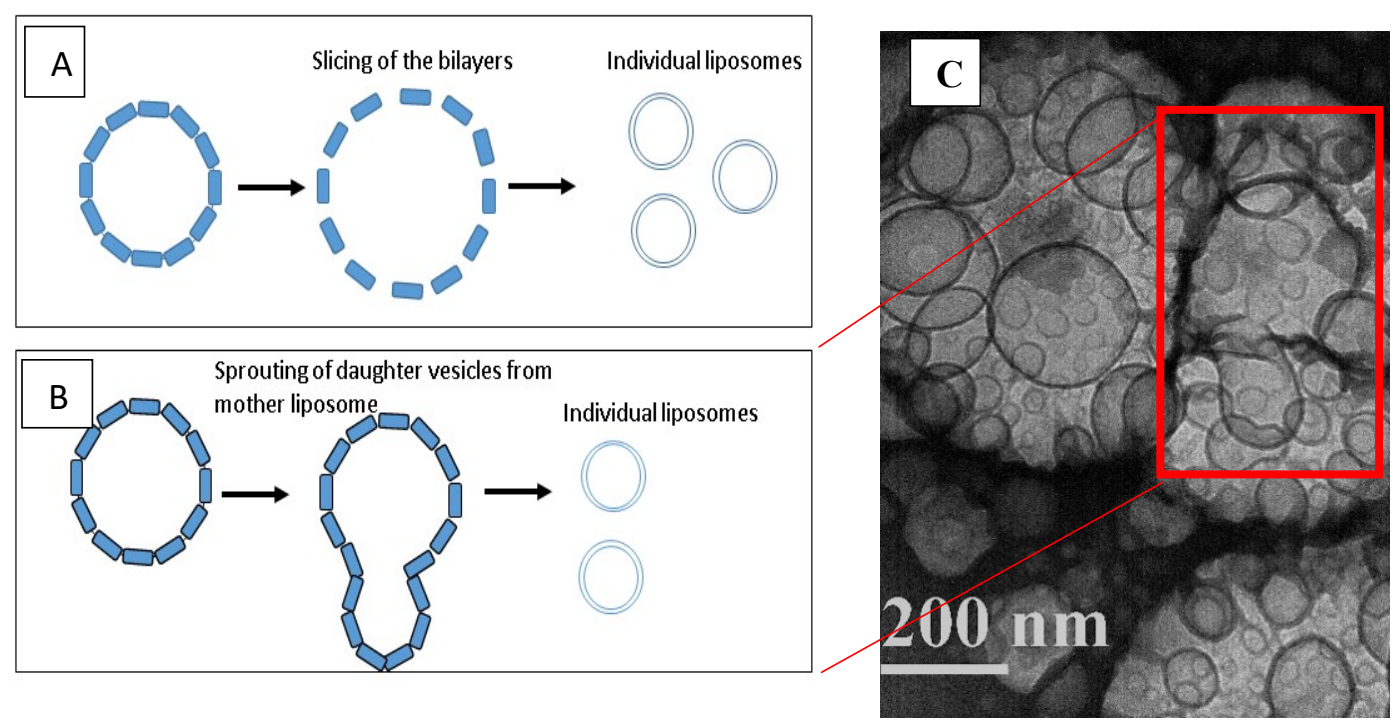

Figure 1: Shows the two proposed mechanism (A) formation of liposomes as the individual bilayer slices form liposomes (B) sprouting of daughter vesicles from mother liposomes (C) Liposomes in GLCs which depicts the mechanism of liposome formation ( Scale: 200nm) 